\title{
Design High Efficiency-Minimum Rule Base PID Like Fuzzy Computed Torque Controller
}

\author{
Alireza Khalilian, Ghasem Sahamijoo, Omid Avatefipour, \\ Farzin Piltan, Mahmoud Reza Safaei Nasrabad \\ Research and Development Department, Institute of Advance Science and Technology-SSP, Shiraz, Iran \\ Email: SSP.ROBOTIC@iranssp.com, WWW.IRANSSP.COM
}

\begin{abstract}
The minimum rule base Proportional Integral Derivative (PID) Fuzzy Computed Torque Controller is presented in this research. The popularity of PID Fuzzy Computed Torque Controller can be attributed to their robust performance in a wide range of operating conditions and partly to their functional simplicity. The process of setting of PID Fuzzy Computed Torque Controller can be determined as an optimization task. Over the years, use of intelligent strategies for tuning of these controllers has been growing. PID methodology has three inputs and if any input is described with seven linguistic values, and any rule has three conditions we will need 343 rules. It is too much work to write 343 rules. In this research the PID-like fuzzy controller can be constructed as a parallel structure of a PD-like fuzzy controller and a PI controller to have the minimum rule base. However computed torque controller is work based on cancelling decoupling and nonlinear terms of dynamic parameters of each link, this controller is work based on manipulator dynamic model and this technique is highly sensitive to the knowledge of all parameters of nonlinear robot manipulator's dynamic equation. This research is used to reduce or eliminate the computed torque controller problem based on minimum rule base fuzzy logic theory to control of flexible robot manipulator system and testing of the quality of process control in the simulation environment of MATLAB/SIMULINK Simulator.
\end{abstract}

Index Terms - PID like fuzzy control, computed torque controller, PD like fuzzy control, PI control, flexible robot manipulator

\section{INTRODUCTION}

Continuum robots represent a class of robots that have a biologically inspired form characterized by flexible backbones and high degrees-of-freedom structures [1-3]. Theoretically, the compliant nature of a continuum robot provides infinite degrees of freedom to these devices. However, there is a limitation set by the practical inability to incorporate infinite actuators in the device. Most of these robots are consequently under actuated (in terms of numbers of independent actuators) with respect to their anticipated tasks. In other words they must achieve a wide range of configurations with relatively few control inputs. This is partly due to the desire to keep the body structures (which, unlike in conventional rigidlink manipulators or fingers, are required to directly contact the environment) "clean and soft", but also to exploit the extra control authority available due to the continuum contact conditions with a minimum number of actuators. For example, the Octarm VI continuum manipulator, discussed frequently in this paper, has nine independent actuated degrees-of-freedom with only three sections. Continuum manipulators differ fundamentally from rigid-link and hyper-redundant robots by having an unconventional structure that lacks links and joints. Hence, standard techniques like the Denavit-Hartenberg (D-H) algorithm cannot be directly applied for developing continuum arm kinematics. Moreover, the design of each continuum arm varies with respect to the flexible backbone present in the system, the positioning, type and number of actuators. The constraints imposed by these factors make the set of reachable configurations and nature of movements unique to every continuum robot. This makes it difficult to formulate generalized kinematic or dynamic models for continuum robot hardware. Thus, the kinematics (i.e. geometry based modeling) of a quite general set of prototypes of continuum manipulators has been developed and basic control strategies now exist based on these. The development of analytical models to analyze continuum arm dynamics (i.e. physics based models involving forces in addition to geometry) is an active, ongoing research topic in this field. From a practical perspective, the modeling approaches currently available in the literature prove to be very complicated and a dynamic model which could be conveniently implemented in an actual device's real-time controller has not been developed yet. The absence of a computationally tractable dynamic model for these robots also prevents the study of interaction of external forces and the impact of collisions on these continuum structures. This impedes the study and ultimate usage of continuum robots in various practical applications like grasping and manipulation, where impulsive dynamics [4-10] are important factors. Although continuum robotics is an interesting subclass of robotics with promising applications for the future, from the current state of the literature, this field is still in its stages of inception.

Controller (control system) is a device which can sense information from linear or nonlinear system (e.g., robot arm) to improve the systems performance and the immune system behavior [11-20]. In feedback control 
system considering that there are many disturbances and also variable dynamic parameters something that is really necessary is keeping plant variables close to the desired value. Feedback control system development is the most important thing in many different fields of safety engineering. The main targets in design control systems are safety stability, good disturbance rejection to reach the best safety, and small tracking error[21-33]. At present, in some applications robot arms are used in unknown and unstructured environment, therefore strong mathematical tools used in new control methodologies to design nonlinear robust controller with an acceptable safety performance (e.g., minimum error, good trajectory, disturbance rejection). According to the control theory, systems' controls are divided into two main groups: conventional control theory and soft computing control theory. Conventional control theories are work based on manipulator dynamic model. This technique is highly sensitive to the knowledge of all parameters of nonlinear robot manipulator's dynamic equation. Conventional control theory is divided into two main groups: linear control theory and nonlinear control theory. Soft computing (intelligent) control theory is free of some challenges associated to conventional control theory. This technique is worked based on intelligent control theory. This theory is divided into the following groups: fuzzy logic theory, neural network theory, genetic algorithm and neuro-fuzzy theory.

One of the most important nonlinear safety controllers is computed torque methodology which is used in nonlinear certain systems. This methodology is used in wide range areas such as in safety control access process; in aerospace applications and in robotics because this methodology can solve some main challenging topics in safety control access such as resistivity to the external disturbance and stability. Even though, this methodology is used in wide range areas but, pure computed torque method has an important drawbacks beside uncertain system and also in presence of external disturbance. Uncertainty in system can causes some problems about safety in industrial factory [34-50].

Although the fuzzy-logic control is not a new technique, its application in this current research is considered to be novel since it aimed for an automated dynamic-less response rather than for the traditional objective of uncertainties compensation[38-57]. The intelligent tracking control using the fuzzy-logic technique provides a cost-and-time efficient control implementation due to the automated dynamic-less input. This in turn would further inspire multi-uncertainties testing for continuum robot [38-65].
This method is based on design PID like fuzzy controller based on the minimum rule base and applied this controller to conventional computed torque controller to solve the robust challenge in computed torque controller and have a linear behavior based on the nonlinear control system.

This paper is organized as follows; section 2, is served as an introduction to the dynamic of continuum robot, computed torque controller, design linear PID controller and fuzzy inference system. Part 3, introduces and describes the methodology algorithm. Section 4 presents the simulation results and discussion of this algorithm applied to a continuum robot and the last part is described as conclusion.

\section{THEORY}

Dynamic Formulation of Continuum Robot: The Continuum section analytical model developed here consists of three modules stacked together in series. In general, the model will be a more precise replication of the behavior of a continuum arm with a greater of modules included in series. However, we will show that three modules effectively represent the dynamic behavior of the hardware, so more complex models are not motivated. Thus, the constant curvature bend exhibited by the section is incorporated inherently within the model. The model resulting from the application of Lagrange's equations of motion obtained for this system can be represented in the form

$$
F_{\text {coeff }} \underline{\tau}=D(\underline{q}) \underline{\ddot{q}}+C(\underline{q}) \underline{\dot{q}}+G(\underline{q})
$$

where $\tau$ is a vector of input forces and $\mathrm{q}$ is a vector of generalized co-ordinates. The force coefficient matrix $F_{\text {coeff }}$ transforms the input forces to the generalized forces and torques in the system. The inertia matrix, $D$ is composed of four block matrices. The block matrices that correspond to pure linear accelerations and pure angular accelerations in the system (on the top left and on the bottom right) are symmetric. The matrix $C$ contains coefficients of the first order derivatives of the generalized co-ordinates. Since the system is nonlinear, many elements of $C$ contain first order derivatives of the generalized co-ordinates. The remaining terms in the dynamic equations resulting from gravitational potential energies and spring energies are collected in the matrix $G$. The coefficient matrices of the dynamic equations are given below,

$$
\text { Fcoeff }=\left[\begin{array}{cccccc}
1 & 1 & \cos \left(\theta_{1}\right) & \cos \left(\theta_{1}\right) & \cos \left(\theta_{1}+\theta_{2}\right) & \cos \left(\theta_{1}+\theta_{2}\right) \\
0 & 0 & 1 & 1 & \cos \left(\theta_{2}\right) & \cos \left(\theta_{2}\right) \\
0 & 0 & 0 & 0 & 1 & 1 \\
1 / 2 & -1 / 2 & 1 / 2 & -1 / 2 & 1 / 2+s_{2} \sin \left(\theta_{2}\right) & -1 / 2+s_{2} \sin \left(\theta_{2}\right) \\
0 & 0 & 1 / 2 & -1 / 2 & 1 / 2 & -1 / 2 \\
0 & 0 & 0 & 0 & 1 / 2 & -1 / 2
\end{array}\right]
$$




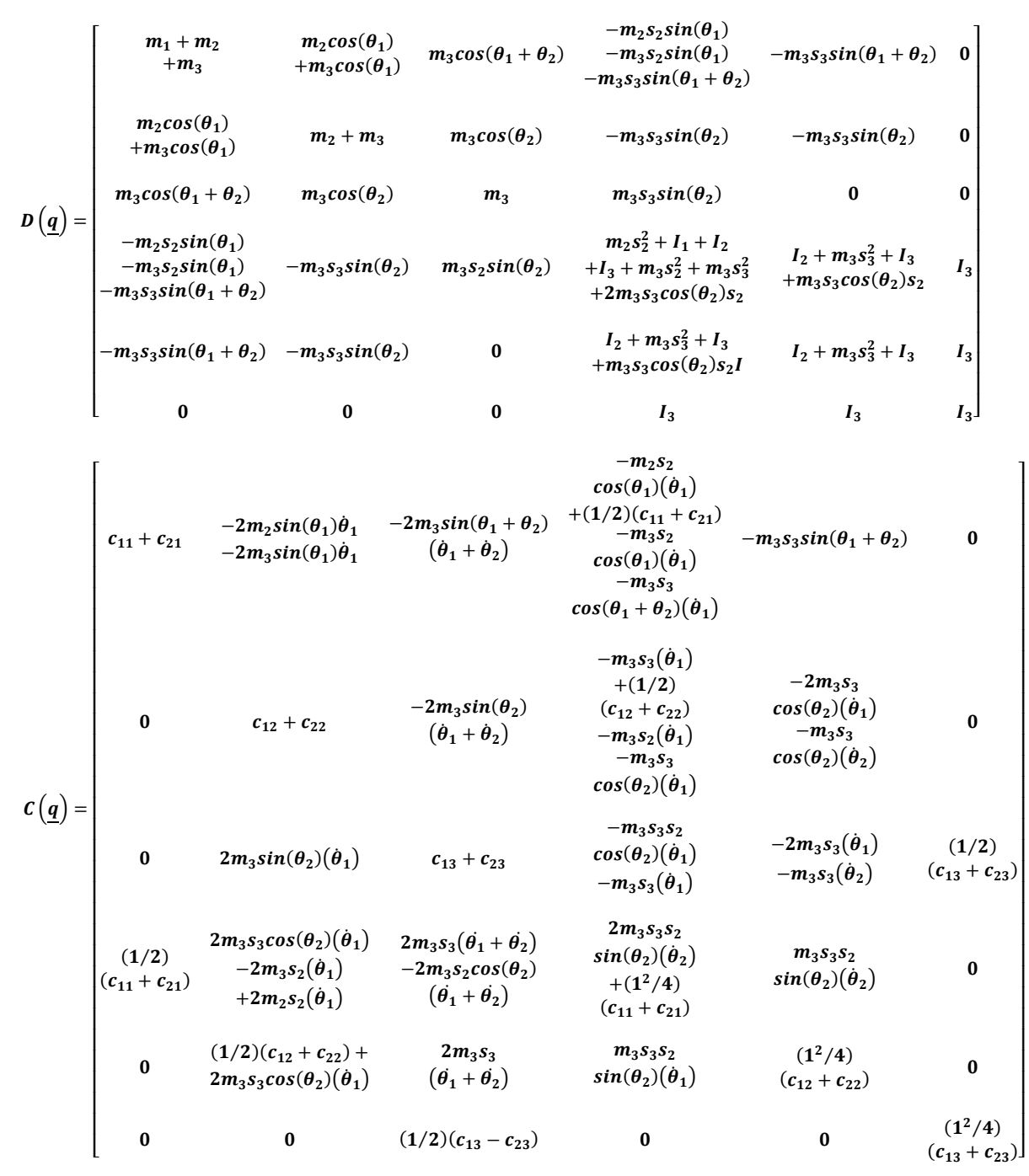

$$
G(\underline{q})=\left[\begin{array}{c}
-m_{1} g-m_{2} g+k_{11}\left(s_{1}+(1 / 2) \theta_{1}-s_{01}\right)+k_{21}\left(s_{1}-(1 / 2) \theta_{1}-s_{01}\right)-m_{3} g \\
-m_{2} g \cos \left(\theta_{1}\right)+k_{12}\left(s_{2}+(1 / 2) \theta_{2}-s_{02}\right)+k_{22}\left(s_{2}-(1 / 2) \theta_{2}-s_{02}\right)-m_{3} g \cos \left(\theta_{1}\right) \\
-m_{3} g \cos \left(\theta_{1}+\theta_{2}\right)+k_{13}\left(s_{3}+(1 / 2) \theta_{3}-s_{03}\right)+k_{23}\left(s_{3}-(1 / 2) \theta_{3}-s_{03}\right) \\
m_{2} s_{2} g \sin \left(\theta_{1}\right)+m_{3} s_{3} g \sin \left(\theta_{1}+\theta_{2}\right)+m_{3} s_{2} g \sin \left(\theta_{1}\right)+k_{11}\left(s_{1}+(1 / 2) \theta_{1}-s_{01}\right)(1 / 2) \\
+k_{21}\left(s_{1}-(1 / 2) \theta_{1}-s_{01}\right)(-1 / 2) \\
m_{3} s_{3} g \sin \left(\theta_{1}+\theta_{2}\right)+k_{12}\left(s_{2}+(1 / 2) \theta_{2}-s_{02}\right)(1 / 2)+k_{22}\left(s_{2}-(1 / 2) \theta_{2}-s_{02}\right)(-1 / 2) \\
k_{13}\left(s_{3}+(1 / 2) \theta_{3}-s_{03}\right)(1 / 2)+k_{23}\left(s_{3}-(1 / 2) \theta_{3}-s_{03}\right)(-1 / 2)
\end{array}\right]
$$

Computed torque methodology: Computed torque controller (CTC) is a powerful nonlinear controller which it widely used in control of nonlinear systems. It is based on feedback linearization and computes the required arm torques using the nonlinear feedback control law. This controller works very well when all dynamic and physical parameters are known but when the robot has variation in dynamic parameters, in this situation the controller has no acceptable trajectory performance[14]. In practice, most of physical systems (e.g., continuum robot) parameters are unknown or time variant, therefore, computed torque like controller used to compensate dynamic equation of continuum robot [13-23]. When all dynamic and physical parameters are known, computed torque controller works fantastically; practically a large amount of systems have uncertainties, therefore computed torque like controller is the best case to solve this challenge. The central idea of computed torque controller (CTC) is feedback linearization so, originally this algorithm is called feedback linearization controller. It has assumed that the desired motion trajectory for the manipulator $\boldsymbol{q}_{\boldsymbol{d}}(\boldsymbol{t})$, as determined, by a path planner. Defines the tracking error as:

$$
e(t)=q_{d}(t)-q_{a}(t)
$$

Where e(t) is error of the plant, $\boldsymbol{q}_{\boldsymbol{d}}(\boldsymbol{t})$ is desired input variable, that in our system is desired displacement, $\boldsymbol{q}_{\boldsymbol{a}}(\boldsymbol{t})$ is actual displacement. If an alternative linear state- 
space equation in the form $\dot{\boldsymbol{x}}=\boldsymbol{A} \boldsymbol{x}+\boldsymbol{B} \boldsymbol{U}$ can be defined as

$$
\dot{x}=\left[\begin{array}{ll}
\mathbf{0} & I \\
\mathbf{0} & \mathbf{0}
\end{array}\right] \boldsymbol{x}+\left[\begin{array}{l}
\mathbf{0} \\
\boldsymbol{I}
\end{array}\right] \boldsymbol{U}
$$

With $\boldsymbol{U}=-\boldsymbol{D}^{-\mathbf{1}}(\boldsymbol{q}) \cdot \boldsymbol{N}(\boldsymbol{q}, \dot{\boldsymbol{q}})+\boldsymbol{D}^{-\mathbf{1}}(\boldsymbol{q}) \cdot \boldsymbol{\tau}$ and this is known as the Brunousky canonical form. By equation (6) and (7) the Brunousky canonical form can be written in terms of the state $\boldsymbol{x}=\left[\boldsymbol{e}^{T} \dot{e}^{T}\right]^{T}$ as [24]:

$$
\frac{d}{d t}\left[\begin{array}{l}
e \\
\dot{e}
\end{array}\right]=\left[\begin{array}{cc}
0 & I \\
0 & 0
\end{array}\right] \cdot\left[\begin{array}{l}
e \\
\dot{e}
\end{array}\right]+\left[\begin{array}{l}
0 \\
I
\end{array}\right] U
$$

With

$$
U=\ddot{q}_{d}+D^{-1}(q) \cdot\{N(q \cdot \dot{q})-\tau\}
$$

Then compute the required arm torques using inverse of equation (32), is;

$$
\tau=D(q)\left(\ddot{q_{d}}-U\right)+N(\dot{q}, q)
$$

This is a nonlinear feedback control law that guarantees tracking of desired trajectory. Selecting proportional-plus-derivative (PD) feedback for $\mathrm{U}(\mathrm{t})$ results in the PD-computed torque controller [24];

$$
\tau=D(q)\left(\ddot{q}_{d}+K_{v} \dot{e}+K_{p} e\right)+N(q, \dot{q})
$$

and the resulting linear error dynamics are

$$
\left(\ddot{q}_{d}+K_{v} \dot{e}+K_{p} e\right)=0
$$

According to the linear system theory, convergence of the tracking error to zero is guaranteed [24]. Where $\boldsymbol{K}_{\boldsymbol{p}}$ and $\boldsymbol{K}_{\boldsymbol{v}}$ are the controller gains. Figure 1 shows the computed torque controller for $6 \mathrm{DOF}$ continuum robot manipulator.

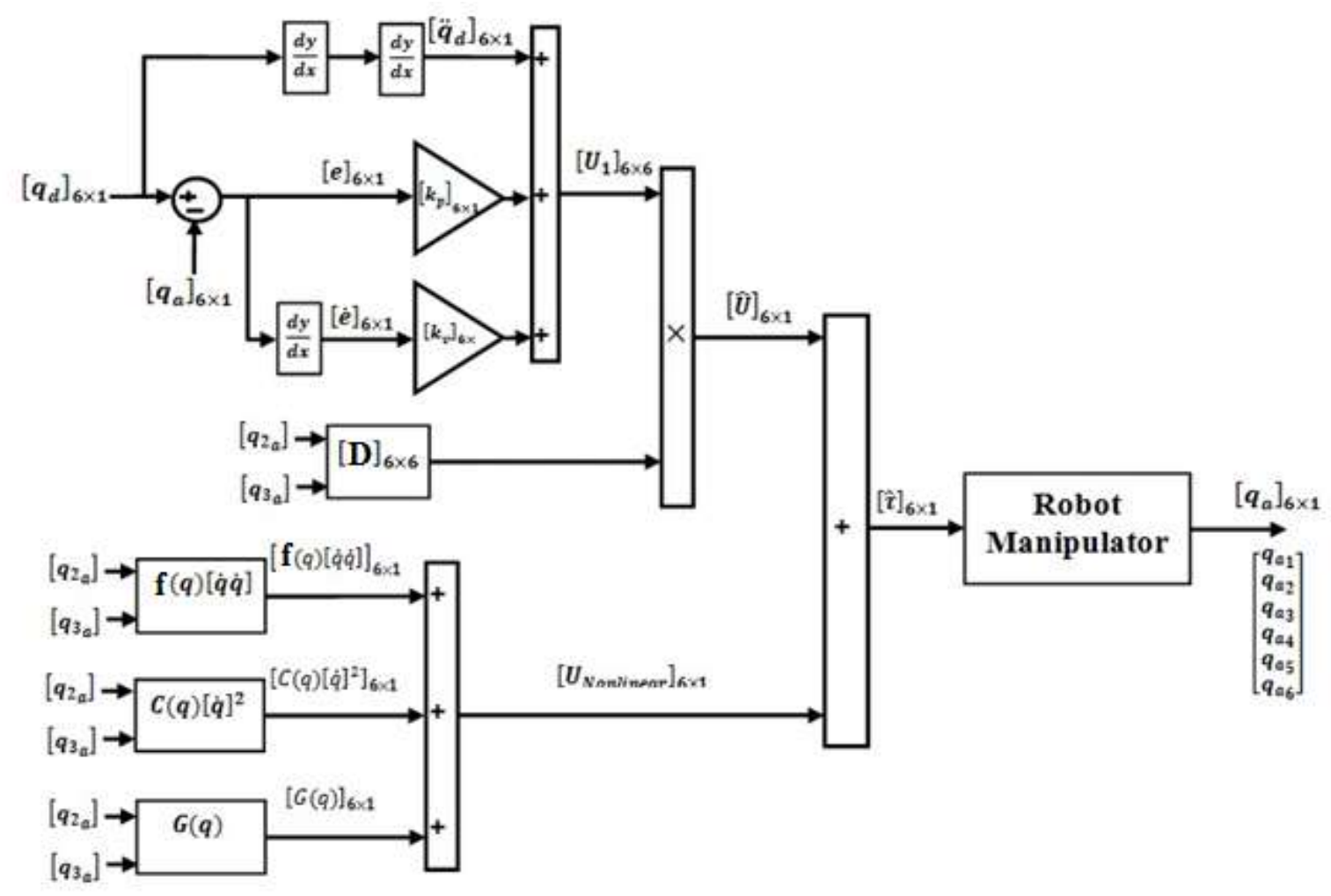

Fig. 1. Computed Torque Control: Continuum Robot Manipulator

Fuzzy Logic Controller: Based on foundation of fuzzy logic methodology; fuzzy logic controller has played important rule to design nonlinear controller for nonlinear and uncertain systems [33]. However the application area for fuzzy control is really wide, the basic form for all command types of controllers consists of;

Input fuzzification (binary-to-fuzzy $[\mathrm{B} / \mathrm{F}]$ conversion)

Fuzzy rule base (knowledge base), Inference engine and Output defuzzification (fuzzy-to-binary [F/B] conversion). Figure 2shows a fuzzy controller block diagram.

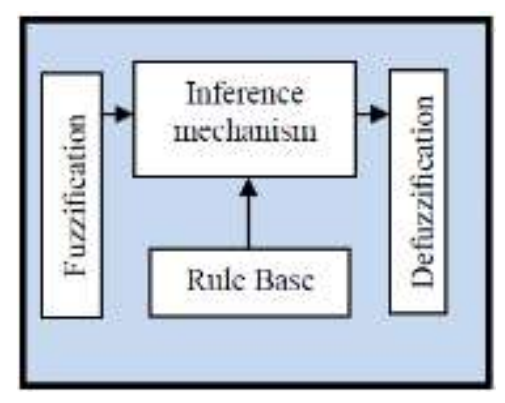

Fig. 2. Fuzzy Controller Part 
The fuzzy inference engine offers a mechanism for transferring the rule base in fuzzy set which it is divided into two most important methods, namely, Mamdani method and Sugeno method. Mamdani method is one of the common fuzzy inference systems and he designed one of the first fuzzy controllers to control of system engine. Mamdani's fuzzy inference system is divided into four major steps: fuzzification, rule evaluation, aggregation of the rule outputs and defuzzification. Michio Sugeno use a singleton as a membership function of the rule consequent part. The following definition shows the Mamdani and Sugeno fuzzy rule base

\section{if $x$ is $A$ and $y$ is $B$ then $z$ is C 'mamdani' \\ if $x$ is $A$ and $y$ is $B$ then $z$ is $f(x, y)$ 'sugeno'}

When $x$ and $y$ have crisp values fuzzification calculates the membership degrees for antecedent part. Rule evaluation focuses on fuzzy operation $(A N D / O R)$ in the antecedent of the fuzzy rules. The aggregation is used to calculate the output fuzzy set and several methodologies can be used in fuzzy logic controller aggregation, namely, Max-Min aggregation, Sum-Min aggregation, Max-bounded product, Max-drastic product, Max-bounded sum, Max-algebraic sum and Min-max. Two most common methods that used in fuzzy logic controllers are Max-min aggregation and Sum-min aggregation. Max-min aggregation defined as below;

$$
\begin{aligned}
& \mu_{U}\left(x_{k}, y_{k}, U\right)=\mu_{\cup_{i=1}^{r} F R^{i}}\left(x_{k}, y_{k}, U\right) \\
& =\max \left\{\min _{i=1}^{r}\left[\mu_{R}\left(x_{k q}, y_{k}\right), \mu_{p_{m}}(U)\right]\right\}
\end{aligned}
$$

The Sum-min aggregation defined as below

$$
\begin{aligned}
& \mu_{U}\left(x_{k}, y_{k}, U\right)=\mu_{\cup i=1}^{r} F R^{i}\left(x_{k}, y_{k}, U\right) \\
& =\sum \min _{i=1}^{r}\left[\mu_{R p q}\left(x_{k}, y_{k}\right), \mu_{p_{m}}(U)\right]
\end{aligned}
$$

where $r$ is the number of fuzzy rules activated by $x_{k}$ and $y_{k}$ and also $\mu_{\cup_{i=1}^{r} F R^{i}}\left(x_{k}, y_{k}, U\right)$ is a fuzzy interpretation of $i-t h$ rule. Defuzzification is the last step in the fuzzy inference system which it is used to transform fuzzy set to crisp set. Consequently defuzzification's input is the aggregate output and the defuzzification's output is a crisp number. Centre of gravity method $(C O G)$ and Centre of area method $(C O A)$ are two most common defuzzification methods, which COG method used the following equation to calculate the defuzzification

$$
\operatorname{COG}\left(x_{k}, y_{k}\right)=\frac{\sum_{i} U_{i} \sum_{j=1}^{r} \cdot \mu_{u}\left(x_{k}, y_{k}, U_{i}\right)}{\sum_{i} \sum_{j=1}^{r} \cdot \mu_{u}\left(x_{k}, y_{k}, U_{i}\right)}
$$

and $C O A$ method used the following equation to calculate the defuzzification

$$
\operatorname{COA}\left(x_{k}, y_{k}\right)=\frac{\sum_{i} U_{i} \cdot \mu_{u}\left(x_{k}, y_{k}, U_{i}\right)}{\sum_{i} \mu_{U} \cdot\left(x_{k}, y_{k}, U_{i}\right)}
$$

Where $\operatorname{COG}\left(x_{k}, y_{k}\right)$ and $\operatorname{COA}\left(x_{k}, y_{k}\right)$ illustrates the crisp value of defuzzification output, $U_{i} \in U$ is discrete element of an output of the fuzzy set, $\mu_{U} \cdot\left(x_{k}, y_{k}, U_{i}\right)$ is the fuzzy set membership function, and $r$ is the number of fuzzy rules.

Design PID Controller: Design of a linear methodology to control of continuum robot manipulator was very straight forward. Since there was an output from the torque model, this means that there would be two inputs into the PID controller. Similarly, the outputs of the controller result from the two control inputs of the torque signal. In a typical PID method, the controller corrects the error between the desired input value and the measured value. Since the actual position is the measured signal. Figure 3 shows linear PID methodology, applied to continuum robot manipulator [21-34].

$$
\begin{aligned}
& e(t)=\theta_{a}(t)-\theta_{d}(t) \\
& U_{P I D}=K_{p_{a}} e+K_{V_{a}} \dot{e}+K_{I} \sum e
\end{aligned}
$$

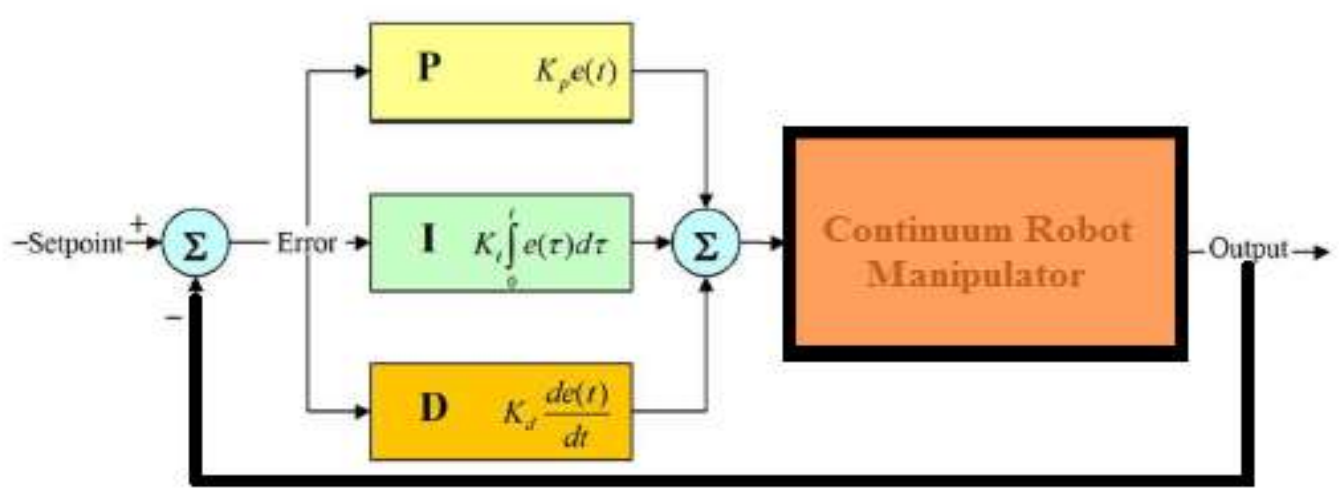

Fig. 3. Block diagram of linear PID method

The model-free control strategy is based on the assumption that the joints of the manipulators are all independent and the system can be decoupled into a group of single-axis control systems [18-23]. Therefore, 
the kinematic control method always results in a group of individual controllers, each for an active joint of the manipulator. With the independent joint assumption, no a priori knowledge of robot manipulator dynamics is needed in the kinematic controller design, so the complex computation of its dynamics can be avoided and the controller design can be greatly simplified. This is suitable for real-time control applications when powerful processors, which can execute complex algorithms rapidly, are not accessible. However, since joints coupling is neglected, control performance degrades as operating speed increases and a manipulator controlled in this way is only appropriate for relatively slow motion $[44,46]$. The fast motion requirement results in even higher dynamic coupling between the various robot joints, which cannot be compensated for by a standard robot controller such as PID [50], and hence model-based control becomes the alternative.

\section{Methodology}

Conversely pure computed torque controller is a highquality nonlinear controller; it has an important problem; nonlinear equivalent dynamic formulation in uncertain dynamic parameter. Computed torque controller is a nonlinear controller but it has a challenge in stability and robustness especially in presence of uncertainty and disturbance. Based on literature CTC formulation is written by;

$$
\tau=D(q)\left(\ddot{q}_{d}+K_{v} \dot{e}+K_{p} e\right)+N(q, \dot{q})
$$

The main challenge in this research is the role of nonlinearity term in presence of uncertainty. To solve this main challenge artificial intelligence based controller is introduce. This type of controller is intelligent therefore design a dynamic of system based on experience knowledge is done by this method. One of the main artificial intelligence techniques is fuzzy logic theory. In this theory the behavior and dynamic of controller is defined by rule base. However defined and number of rule base play important role to design high quality controller but system has limitation to the number of rule base to implementation and the speed of response. Based on literature PID controller can reduce or eliminate the steady state error and design stable controller. But this type of controller has three types of inputs; proportional part, integral part and derivative part. To design PID like fuzzy controller and if any input is described with seven linguistic values, and any rule has three conditions we will need $7 \times 7 \times 7=343$ rules. It is too much work to write 343 rules, the speed of system is too low and design embedded controller based on FPGA or CPLD is very difficult. Based on (9) the PID controller has three inputs and three coefficients. In PD like fuzzy controller error and change of error are the inputs and if any input is described with seven linguistic values, and any rule has two conditions we will need $7 \times 7=49$ rules. Table 1 shows the rule table of PD like fuzzy controller based on seven linguistic variables for inputs and totally 49 rules.
Table 1. Rule Table of PD like Fuzzy Controller

\begin{tabular}{|c|c|c|c|c|c|c|c|}
\hline$e^{\Delta} \Delta e^{\prime}$ & $\mathrm{PB}$ & PM & PS & z & NS & $\mathrm{NM}$ & NB \\
\hline PB & $\mathbf{N B}$ & NB & NB & NB & NM & NS & $\mathbf{Z}$ \\
\hline PM & $\mathrm{NB}$ & $\mathrm{NB}$ & NB & NMI & NS & Z & PS \\
\hline PS & NB & NB & NM & NS & Z & PS & PM \\
\hline $\mathbf{Z}$ & NB & NM & NS & $\mathbf{Z}$ & PS & PM & PB \\
\hline NS & NM & Ns & Z & PS & PM & PB & PB \\
\hline NM & NS & Z & PS & PM & PB & PB & PB \\
\hline NB & $\mathbf{Z}$ & PS & PM & PB & PB & PB & PB \\
\hline
\end{tabular}

This table includes 49 rules. We are taking into account now not just the error but the change-of-error as well. It allows describing the dynamics of the controller. To explain how this rules set works and how to choose the rules, let us divide the set of all rules into the following five groups:

Group 1: In this group of rules both $\boldsymbol{e}$ and $\boldsymbol{\Delta} \boldsymbol{e}$ are (positive or negative) small or zero. This means that the current value of the process output variable has deviated from the desired level (the set-point) but is still close to it. Because of this closeness the control signal should be zero or small in magnitude and is intended to correct small deviations from the set-point. Therefore, the rules in this group are related to the steady-state behavior of the process. The change-of-error, when it is Negative Small or Positive Small, shifts the output to negative or positive region, because in this case, for example, when $\boldsymbol{e}(\boldsymbol{t})$ and $\Delta \boldsymbol{e}(\boldsymbol{t})$ are both Negative Small the error is already negative and, due to the negative change-of-error, tends to become more negative. To prevent this trend, one needs to increase the magnitude of the control output.

Group 2: For this group of rules $\boldsymbol{e}(\boldsymbol{t})$ is Positive Big or Medium which implies that actual input is significantly above the set point. At the same time since $\Delta \boldsymbol{e}(\boldsymbol{t})$ is negative, this means that actual input is moving towards the set-point. The control signal is intended to either speed up or slow down the approach to the set-point. For example, if actual input is much below the set-point $(\boldsymbol{e}(\boldsymbol{t})$ is Positive Big) and it is moving towards the set-point with a small step $(\boldsymbol{\Delta e}(\boldsymbol{t})$ is Negative Small) then the magnitude of this step has to be significantly increased ( $\boldsymbol{U}$ is Negative Medium). However, when actual input is still much below the set-point $(\boldsymbol{e}(\boldsymbol{t})$ is Positive Big) but it is moving towards the set-point very fast $(\boldsymbol{\Delta e}(\boldsymbol{t})$ is Negative Big) no control action can be recommended because the error will be compensated due to the current trend.

Group 3: For this group of rules actual output is either close to the set-point ( $\boldsymbol{e}(\boldsymbol{t})$ is Positive Small, Zero, Negative Small) or significantly above it (Negative Medium, Negative Big). At the same time, since $\Delta \boldsymbol{e}(\boldsymbol{t})$ is negative, actual input is moving away from the setpoint. The control here is intended to reverse this trend and make actual input, instead of moving away from the set-point, start moving towards it. So here the main 
reason for the control action choice is not just the current error but the trend in its change.

Group 4: For this group of rules $\boldsymbol{e}(\boldsymbol{t})$ is Negative Medium or Big, which means that actual input is significantly below the set-point. At the same time, since $\boldsymbol{\Delta} \boldsymbol{e}(\boldsymbol{t})$ is positive, actual input is moving towards the setpoint. The control is intended to either speed up or slow down the approach to the set-point. For example, if actual input is much above the set-point $(\boldsymbol{e}(\boldsymbol{t})$ is Negative Big) and it is moving towards the set-point with a somewhat large step $(\boldsymbol{\Delta} \boldsymbol{e}(\boldsymbol{t})$ is Positive Medium), then the magnitude of this step has to be only slightly enlarged (output is Negative Small).

Group 5: The situation here is similar to the Group 3 in some sense. For this group of rules $\boldsymbol{e}(\boldsymbol{t})$ is either close to the set-point (Positive Small, Zero, Negative Small) or significantly above it (Positive Medium, Positive Big). At the same time since $\boldsymbol{\Delta} \boldsymbol{e}(\boldsymbol{t})$ is positive actual input is moving away from the set-point. This control signal is intended to reverse this trend and make actual input instead of moving away from the set-point start moving towards it. The PD like fuzzy controller shows in Figure 4.

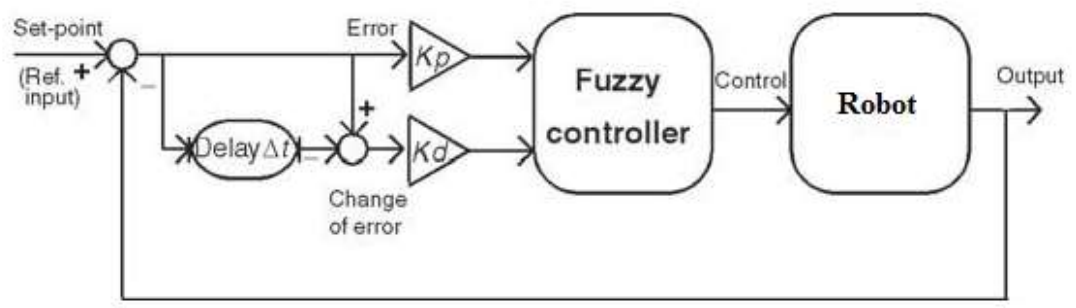

Fig. 4. Block diagram of PD like Fuzzy Controller

We can move the integration from the part proceeding to a fuzzy controller to the part following it. We can integrate the output of a controller, not the input. Based on above discussion the PID-like fuzzy controller can be constructed as a parallel structure of a PD-like fuzzy controller and a PI- controller with the output approximated as:

$$
\begin{aligned}
U_{P I D}=\left(\frac{K_{p_{a}}}{2} e+\right. & \left.K_{V_{a}} \dot{e}\right) \\
& +\left(\frac{K_{p_{a}}}{2} e+K_{I} \sum e\right)
\end{aligned}
$$

In this type of design, we have 49 rule bases for PD like fuzzy controller. This PID like fuzzy controller applied to pure computed torque controller to remove the challenge in this conventional nonlinear controller. Figure 5 shows the block diagram of PID like fuzzy computed torque controller.

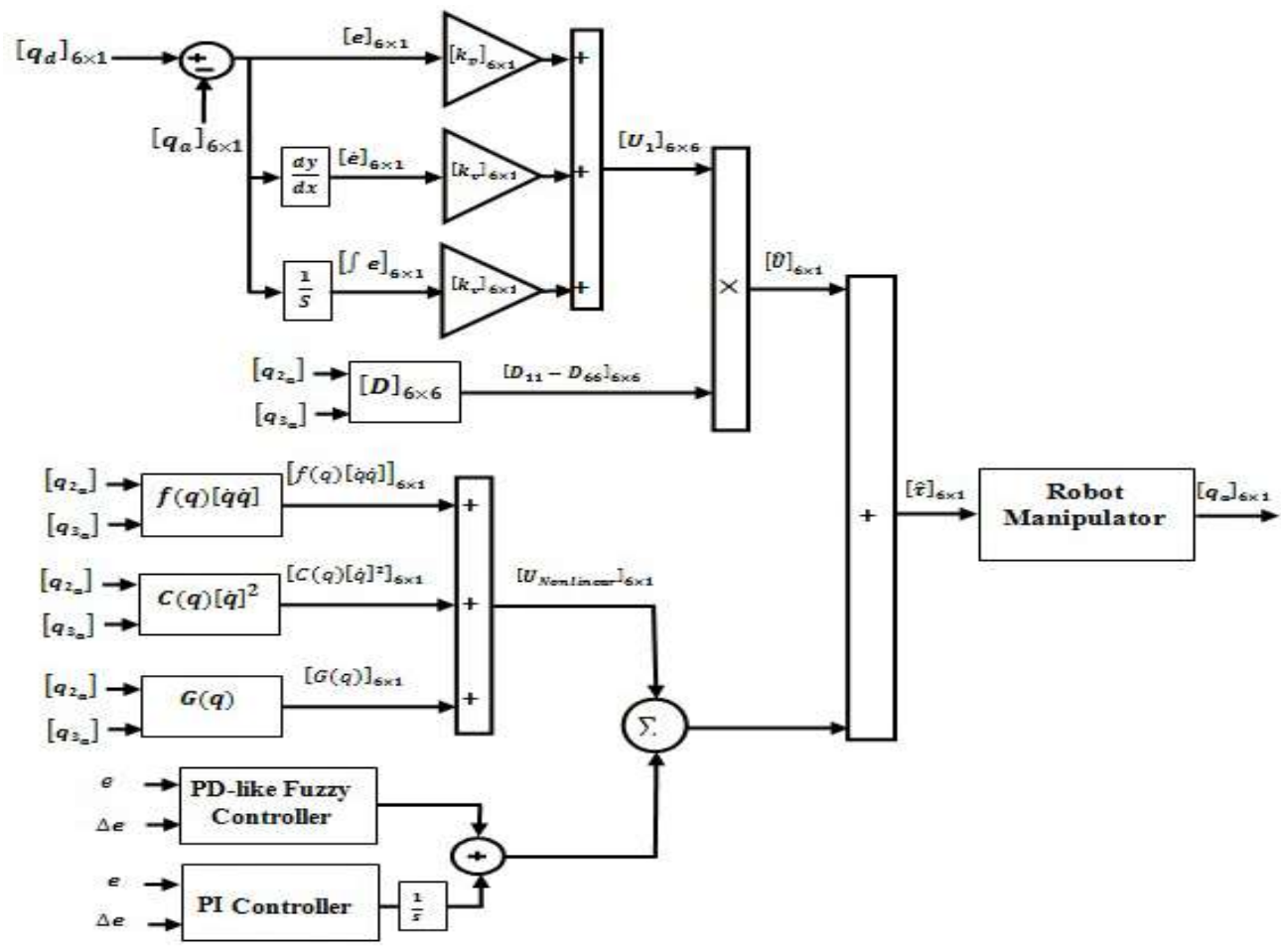

Fig. 5. Block diagram of PID like Fuzzy Computed Torque Controller 


\section{RESUlts AND DisCUSSION}

PID like fuzzy computed torque controller was tested to Step response trajectory. In this simulation, to control position of continuum robot the first, second, and third joints are moved from home to final position without and with external disturbance. The simulation was implemented in MATLAB/SIMULINK environment. These systems are tested by band limited white noise with a predefined $40 \%$ of relative to the input signal amplitude. This type of noise is used to external disturbance in continuous and hybrid systems and applied to nonlinear dynamic of these controllers.

Tracking performances: In proposed controller; nonlinear equivalent part and PID like fuzzy controller are the main part. According to above discussion PID like fuzzy computed torque controller and pure computed torque controller have the same performance in certain system. Based on Fig 6, pure computed torque controller has a slight oscillation therefore in proposed method to solve this challenge the output gain updating factor of PID like fuzzy computed torque controller is decreased. Both of these controllers have the same overshoot and it is about $5 \%$.

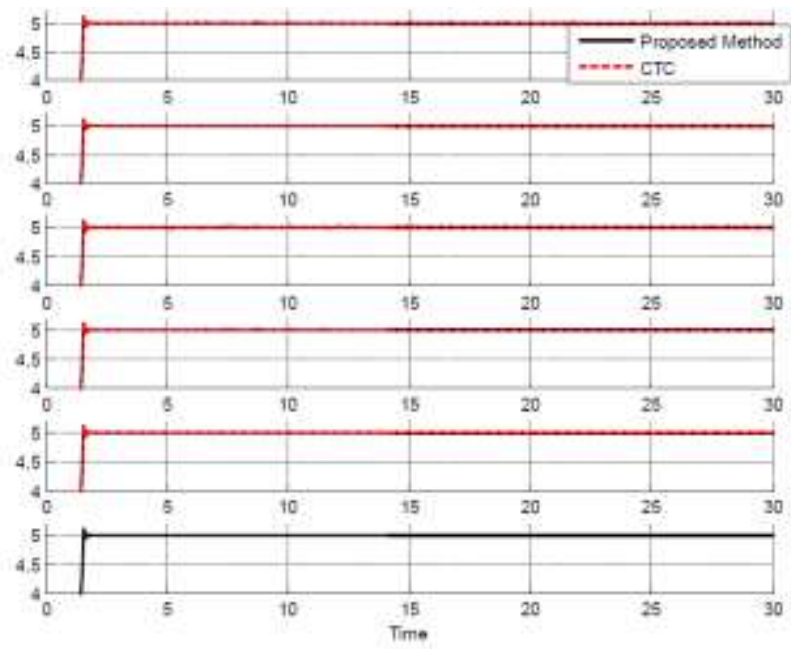

Fig. 6. PID like fuzzy computed torque controller and computed torque controller trajectory following

Disturbance rejection: Figure 7 shows the power disturbance elimination in proposed method and pure computed torque controller in presence of external disturbance and uncertainty parameters. The disturbance rejection is used to test and analyzed the robustness comparisons of these controllers for step trajectory. A band limited white noise with predefined of $40 \%$ the power of input signal value is applied to the step trajectory. It found fairly fluctuations in trajectory responses. According to the following formulation, pure computed torque controller has moderate fluctuation in presence of external disturbance and uncertainty. Pure computed torque controller is unstable. To eliminate this challenge in PID like fuzzy computed torque controller this research is used following methods; decrease the output scaling factor of the PD-part, increase the scaling factor for an integral input compared to other inputs, apply the centre of gravity defuzzification method, reduce the width of the membership function for the zero class of the error signal and Redistribute the membership functions, increasing their concentration around the zero point.

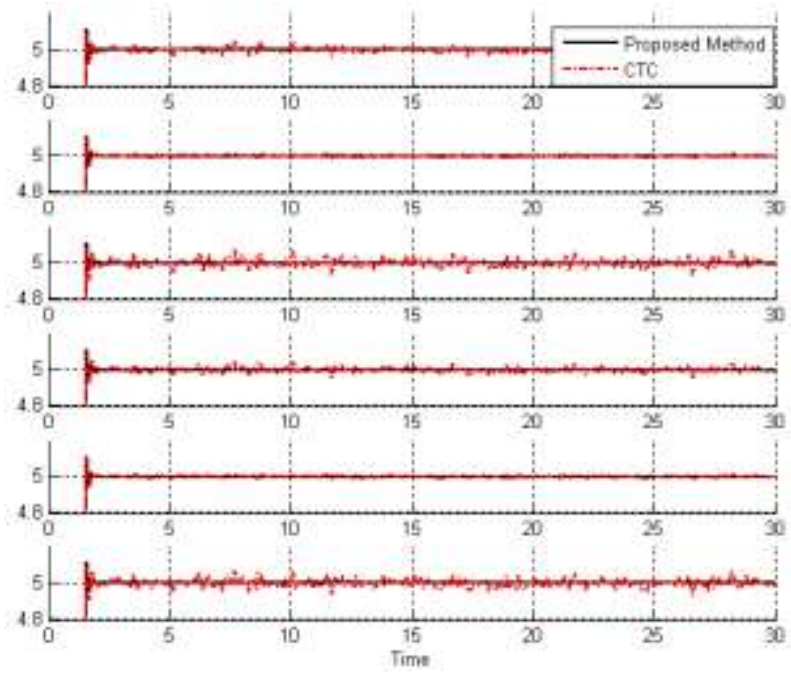

Fig. 7. PID like fuzzy computed torque controller and computed torque controller trajectory following in presence of disturbance

\section{CONCLUSION}

The central issues and challenges of non linear control and estimation problems are to satisfy the desired performance objectives in the presence of noises, disturbances, parameter perturbations, un-modeled dynamics, sensor failures, actuator failures and time delays. Evaluation algorithm PID like fuzzy computed torque controller has shown growing popularity in both industry and academia. To improve the optimality and robustness, we have proposed PD like fuzzy controller parallel with PI like fuzzy controller based on 49 rule base and have one rule table for nonlinear systems with general performance criteria. Computed torque controller provides us an effective tool to control nonlinear systems through the dynamic formulation of nonlinear system. Fuzzy logic controller is used to estimate highly nonlinear dynamic parameters. Mixed performance criteria have been used to design the controller and the relative weighting matrices of these criteria can be achieved by choosing different coefficient matrices. The simulation studies show that the proposed method provides a satisfactory alternative to the existing nonlinear control approaches.

\section{ACKNOWLEDGMENT}

The authors would like to thank the anonymous reviewers for their careful reading of this paper and for their helpful comments. This work was supported by the SSP Institute of Advance Science and Technology Program of Iran under grant no. 2013-Persian Gulf-2A. 


\section{REFERENCES}

[1] T. R. Kurfess, Robotics and automation handbook: CRC, 2005.

[2] J. J. E. Slotine and W. Li, Applied nonlinear control vol. 461: Prentice hall Englewood Cliffs, NJ, 1991.

[3] L. Cheng, et al., "Multi-agent based adaptive consensus control for multiple manipulators with kinematic uncertainties," 2008, pp. 189-194.

[4] J. J. D'Azzo, et al., Linear control system analysis and design with MATLAB: CRC, 2003.

[5] B. Siciliano and O. Khatib, Springer handbook of robotics: Springer-Verlag New York Inc, 2008.

[6] I. Boiko, et al., "Analysis of chattering in systems with second-order sliding modes," IEEE Transactions on Automatic Control, vol. 52, pp. 2085-2102, 2007.

[7] J. Wang, et al., "Indirect adaptive fuzzy sliding mode control: Part I: fuzzy switching," Fuzzy Sets and Systems, vol. 122, pp. 21-30, 2001.

[8] F. Piltan, et al., "Artificial Control of Nonlinear Second Order Systems Based on AFGSMC," Australian Journal of Basic and Applied Sciences, 5(6), pp. 509-522, 2011.

[9] V. Utkin, "Variable structure systems with sliding modes," Automatic Control, IEEE Transactions on, vol. 22, pp. 212-222, 2002.

[10] R. A. DeCarlo, et al., "Variable structure control of nonlinear multivariable systems: a tutorial," Proceedings of the IEEE, vol. 76, pp. 212-232, 2002.

[11] K. D. Young, et al., "A control engineer's guide to sliding mode control," 2002, pp. 1-14.

[12] Samira Soltani \& Farzin Piltan, "Design Artificial Nonlinear Controller Based on Computed Torque like Controller with Tunable Gain", World Applied Science Journal (WASJ), 14 (9): 1306-1312, 2011.

[13] Farzin Piltan, Mohammadali Dialame, Abbas Zare \& Ali Badri,"Design Novel Lookup Table Changed Auto Tuning FSMC:Applied to Robot Manipulator", International Journal of Engineering, 6 (1):25-41, 2012

[14] Farzin Piltan, Mohammad Keshavarz, Ali Badri \& Arash Zargari,"Design Novel Nonlinear Controller Applied to RobotManipulator: Design New Feedback Linearization Fuzzy Controller with Minimum Rule Base Tuning Method", International Journal of Robotics and Automation,3 (1):1-12, 2012

[15] Farzin Piltan, Iman Nazari, Sobhan Siamak, Payman Ferdosali,"Methodology of FPGA-Based Mathematical error-Based Tuning Sliding Mode Controller", International Journal of Control and Automation, 5(1), 89118, 2012

[16] Farzin Piltan, Bamdad Boroomand, Arman Jahed \& Hossein Rezaie, "Methodology of Mathematical ErrorBased Tuning Sliding Mode Controller", International Journal of Engineering, 6 (2):96-117, 2012

[17] Farzin Piltan, Sara Emamzadeh, Zahra Hivand, Fatemeh Shahriyari \& Mina Mirazaei, "PUMA-560 Robot Manipulator Position Sliding Mode Control Methods Using MATLAB/SIMULINK and Their Integration into Graduate/Undergraduate Nonlinear Control, Robotics and MATLAB Courses", International Journal of Robotics and Automation, 3(3):106-150, 2012

[18] Farzin Piltan, Ali Hosainpour, Ebrahim Mazlomian, Mohammad Shamsodini, Mohammad H. Yarmahmoudi, "Online Tuning Chattering Free Sliding Mode Fuzzy Control Design: Lyapunov Approach", International Journal of Robotics and Automation, 3(3):77105,2012
[19] Farzin Piltan, Mina Mirzaei, Forouzan Shahriari, Iman Nazari, Sara Emamzadeh, "Design Baseline Computed Torque Controller", International Journal of Engineering, 6(3): 129-141, 2012

[20] Farzin Piltan, Mohammad H. Yarmahmoudi, Mohammad Shamsodini, Ebrahim Mazlomian, Ali Hosainpour, "PUMA-560 Robot Manipulator Position Computed Torque Control Methods Using MATLAB/SIMULINK and Their Integration into Graduate Nonlinear Control and MATLAB Courses", International Journal of Robotics and Automation, 3(3): 167-191, 2012

[21] Farzin Piltan, Hossein Rezaie, Bamdad Boroomand, Arman Jahed, "Design Robust Backstepping on-line Tuning Feedback Linearization Control Applied to IC Engine", International Journal of Advance Science and Technology, 11:40-22, 2012

[22] Farzin Piltan, Mohammad R. Rashidian, Mohammad Shamsodini and Sadeq Allahdadi, Effect of Rule Base on the Fuzzy-Based Tuning Fuzzy Sliding Mode Controller: Applied to $2^{\text {nd }}$ Order Nonlinear System", International Journal of Advanced Science and Technology, 46:39-70, 2012

[23] Farzin Piltan, Arman Jahed, Hossein Rezaie and Bamdad Boroomand, "Methodology of Robust Linear On-line High Speed Tuning for Stable Sliding Mode Controller: Applied to Nonlinear System", International Journal of Control and Automation, 5(3): 217-236, 2012

[24] Farzin Piltan, Bamdad Boroomand, Arman Jahed and Hossein Rezaie, "Performance-Based Adaptive Gradient Descent Optimal Coefficient Fuzzy Sliding Mode Methodology", International Journal of Intelligent Systems and Applications, , vol.4, no.11, pp.40-52, 2012.

[25] Farzin Piltan, Mehdi Akbari, Mojdeh Piran , Mansour Bazregar, ”Design Model Free Switching Gain Scheduling Baseline Controller with Application to Automotive Engine", International Journal of Information Technology and Computer Science, vol.5, no.1, pp.65-73, 2013.DOI: 10.5815/ijitcs.2013.01.07.

[26] Farzin Piltan, Mojdeh Piran , Mansour Bazregar, Mehdi Akbari, "Design High Impact Fuzzy Baseline Variable Structure Methodology to Artificial Adjust Fuel Ratio", International Journal of Intelligent Systems and Applications, vol.5, no.2, pp.59-70, 2013.DOI: 10.5815/ijisa.2013.02.0.

[27] Farzin Piltan, M. Bazregar, M. kamgari, M. Akbari and M. Piran, "Adjust the Fuel Ratio by High Impact Chattering Free Sliding Methodology with Application to Automotive Engine", International Journal of Hybrid Information Technology, 6(1), 2013.

[28] Farzin Piltan, S. Zare , F. ShahryarZadeh, M. Mansoorzadeh, M. kamgari, "Supervised Optimization of Fuel Ratio in IC Engine Based on Design Baseline Computed Fuel Methodology", International Journal of Information Technology and Computer Science, vol.5, no.4, pp.76-84, 2013.DOI: 10.5815/ijitcs.2013.04.09.

[29] Farzin Piltan, M. Mansoorzadeh, S. Zare, F.Shahryarzadeh, M. Akbari, "Artificial Tune of Fuel Ratio: Design a Novel SISO Fuzzy Backstepping Adaptive Variable Structure Control", International Journal of Electrical and Computer Engineering, 3(2), 2013.

[30] M. Bazregar, Farzin Piltan, A. Nabaee and M.M. Ebrahimi, "Parallel Soft Computing Control Optimization Algorithm for Uncertainty Dynamic Systems", International Journal of Advanced Science and Technology, 51, 2013. 
[31] Farzin Piltan, M.H. Yarmahmoudi, M. Mirzaei, S. Emamzadeh, Z. Hivand, "Design Novel Fuzzy Robust Feedback Linearization Control with Application to Robot Manipulator", International Journal of Intelligent Systems and Applications , vol.5, no.5, pp.1-10, 2013.DOI: 10.5815/ijisa.2013.05.01.

[32] Sh. Tayebi Haghighi, S. Soltani, Farzin Piltan, M. kamgari, S. Zare, "Evaluation Performance of IC Engine: Linear Tunable Gain Computed Torque Controller Vs. Sliding Mode Controller", International Journal of Intelligent Systems and Applications, vol.5, no.6, pp.78-88, 2013.DOI: 10.5815/ijisa.2013.06.10.

[33] Amin Jalali, Farzin Piltan, M. Keshtgar, M. Jalali, "Colonial Competitive Optimization Sliding Mode Controller with Application to Robot Manipulator", International Journal of Intelligent Systems and Applications, vol.5, no.7, pp.50-56, 2013. DOI: 10.5815/ijisa.2013.07.07.

[34] Salehi, Farzin Piltan, M. Mousavi, A. Khajeh, M. R. Rashidian, "Intelligent Robust Feed-forward Fuzzy Feedback Linearization Estimation of PID Control with Application to Continuum Robot", International Journal of Information Engineering and Electronic Business, vol.5, no.1, pp.1-16, 2013. DOI: 10.5815/ijieeb.2013.01.01.

[35] Farzin Piltan, M.J. Rafaati, F. Khazaeni, A. Hosainpour, S. Soltani, “A Design High Impact Lyapunov Fuzzy PD-PlusGravity Controller with Application to Rigid Manipulator", International Journal of Information Engineering and Electronic Business, vol.5, no.1, pp.17-25, 2013. DOI: 10.5815/ijieeb.2013.01.02.

[36] Amin Jalali, Farzin Piltan, A. Gavahian, M. Jalali, M. Adibi, "Model-Free Adaptive Fuzzy Sliding Mode Controller Optimized by Particle Swarm for Robot manipulator", International Journal of Information Engineering and Electronic Business, vol.5, no.1, pp.68-78, 2013. DOI: $10.5815 /$ ijieeb.2013.01.08.

[37] Farzin Piltan, F. ShahryarZadeh ,M. Mansoorzadeh ,M. kamgari, S. Zare, "Robust Fuzzy PD Method with Parallel Computed Fuel Ratio Estimation Applied to Automotive Engine“, International Journal of Intelligent Systems and Applications, vol.5, no.8, pp.83-92, 2013. DOI: 10.5815/ijisa.2013.08.10.

[38] Farzin Piltan, A. Nabaee, M.M. Ebrahimi, M. Bazregar, "Design Robust Fuzzy Sliding Mode Control Technique for Robot Manipulator Systems with Modeling Uncertainties", International Journal of Information Technology and Computer Science, vol.5, no.8, pp.123135, 2013. DOI: $10.5815 /$ ijitcs.2013.08.12.

[39] Farzin Piltan, M. Mansoorzadeh, M. Akbari, S. Zare, F. ShahryarZadeh "Management of Environmental Pollution by Intelligent Control of Fuel in an Internal Combustion Engine" Global Journal of Biodiversity Science And Management, 3(1), 2013.

[40] M. M. Ebrahimit Farzin Piltan, M. Bazregar and A.R. Nabaee, "Intelligent Robust Fuzzy-Parallel Optimization Control of a Continuum Robot Manipulator", International Journal of Control and Automation, 6(3), 2013.

[41] O.R. Sadrnia, Farzin Piltan, M. Jafari, M. Eram and M. Shamsodini, "Design PID Estimator Fuzzy plus Backstepping to Control of Uncertain Continuum Robot", International Journal of Hybrid Information Technology, 6(4), 2013.

[42] AminJalali, Farzin Piltan, H. Hashemzadeh, A. Hasiri, M.R Hashemzadeh, "Design Novel Soft Computing Backstepping Controller with Application to Nonlinear Dynamic Uncertain System", International Journal of
Intelligent Systems and Applications, vol.5, no.10, pp.93105, 2013. DOI: 10.5815/ijisa.2013.10.12.

[43] M. Moosavi, M. Eram, A. Khajeh, O. Mahmoudi and Farzin Piltan, "Design New Artificial Intelligence Base Modified PID Hybrid Controller for Highly Nonlinear System", International Journal of Advanced Science and Technology, 57, 2013.

[44] S. Zahmatkesh, Farzin Piltan, K. Heidari, M. Shamsodini, S. Heidari, "Artificial Error Tuning Based on Design a Novel SISO Fuzzy Backstepping Adaptive Variable Structure Control" International Journal of Intelligent Systems and Applications, vol.5, no.11, pp.34-46, 2013. DOI: 10.5815/ijisa.2013.11.04.

[45] S. Heidari, Farzin Piltan, M. Shamsodini, K. Heidari and S. Zahmatkesh, "Design New Nonlinear Controller with Parallel Fuzzy Inference System Compensator to Control of Continuum Robot Manipulator",International Journal of Control and Automation, 6(4), 2013.

[46] FarzinPiltan, M. Kamgari, S. Zare, F. ShahryarZadeh, M. Mansoorzadeh, "Design Novel Model Reference Artificial Intelligence Based Methodology to Optimized Fuel Ratio in IC Engine", International Journal of Information Engineering and Electronic Business, vol.5, no.2, pp.44-51, 2013. DOI: 10.5815/ijieeb.2013.02.07.

[47] Farzin Piltan, Mehdi Eram, Mohammad Taghavi, Omid Reza Sadrnia, Mahdi Jafari,"Nonlinear Fuzzy Model-base Technique to Compensate Highly Nonlinear Continuum Robot Manipulator", IJISA, vol.5, no.12, pp.135-148, 2013. DOI: $10.5815 /$ ijisa.2013.12.12

[48] Amin Jalali, Farzin Piltan, Mohammadreza Hashemzadeh, Fatemeh BibakVaravi, Hossein Hashemzadeh,"Design Parallel Linear PD Compensation by Fuzzy Sliding Compensator for Continuum Robot", IJITCS, vol.5, no.12, pp.97-112, 2013. DOI: 10.5815/ijitcs.2013.12.12

[49] Farzin Piltan, A. Hosainpour, S. Emamzadeh, I. Nazari, M. Mirzaie, "Design Sliding Mode Controller of with Parallel Fuzzy Inference System Compensator to Control of Robot Manipulator", International Journal of Robotics and Automation, Vol. 2, No. 4, December 2013, pp. 149 162.

[50] Farzin Piltan, Mahdi Jafari, Mehdi Eram, Omid Mahmoudi, Omid Reza Sadrnia, "Design Artificial Intelligence-Based Switching PD plus Gravity for Highly Nonlinear Second Order System", International Journal of Engineering and Manufacturing, vol.3, no.1, pp.38-57, 2013.DOI: 10.5815/ijem.2013.01.04

[51] Farzin Piltan, Sara Emamzadeh, Sara Heidari, Samaneh Zahmatkesh, Kamran Heidari, "Design Artificial Intelligent Parallel Feedback Linearization of PID Control with Application to Continuum Robot", International Journal of Engineering and Manufacturing, vol.3, no.2, pp.51-72, 2013.DOI: 10.5815/ijem.2013.02.04

[52] Mohammad Mahdi Ebrahimi, Farzin Piltan, Mansour Bazregar, AliReza Nabaee,"Artificial Chattering Free online Modified Sliding Mode Algorithm: Applied in Continuum Robot Manipulator", International Journal of Information Engineering and Electronic Business, vol.5, no.5, pp.57-69, 2013. DOI: 10.5815/ijieeb.2013.05.08

[53] Arman Jahed, Farzin Piltan, Hossein Rezaie, Bamdad Boroomand, "Design Computed Torque Controller with Parallel Fuzzy Inference System Compensator to Control of Robot Manipulator", International Journal of Information Engineering and Electronic Business, vol.5, no.3, pp.66-77, 2013. DOI: 10.5815/ijieeb.2013.03.08

[54] Mohammad Shamsodini, Farzin Piltan, Mahdi Jafari, Omid reza Sadrnia, Omid Mahmoudi,"Design Modified Fuzzy 
Hybrid Technique: Tuning By GDO", IJMECS, vol.5, no.8, pp.58-72, 2013.DOI: 10.5815/ijmecs.2013.08.07

[55] Mahdi Mirshekaran, Farzin Piltan,Zahra Esmaeili, Tannaz Khajeaian, Meysam Kazeminasab,"Design Sliding Mode Modified Fuzzy Linear Controller with Application to Flexible Robot Manipulator", IJMECS, vol.5, no.10, pp.53-63, 2013.DOI: 10.5815/ijmecs.2013.10.07

[56] Meysam Kazeminasab, Farzin Piltan, Zahra Esmaeili, Mahdi Mirshekaran, Alireza Salehi ,"Design Parallel Fuzzy Partly Inverse Dynamic Method plus Gravity Control for Highly Nonlinear Continuum Robot", IJISA, vol.6, no.1, pp.112-123, 2014. DOI: 10.5815/ijisa.2014.01.12

[57] Mansour Bazregar, Farzin Piltan, Mehdi Akbari, Mojdeh Piran,"Management of Automotive Engine Based on Stable Fuzzy Technique with Parallel Sliding Mode Optimization", IJITCS, vol.6, no.1, pp.101-107, 2014. DOI 10.5815/ijitcs.2014.01.12.

[58] Ali Shahcheraghi, Farzin Piltan, Masoud Mokhtar, Omid Avatefipour, Alireza Khalilian,"Design a Novel SISO Offline Tuning of Modified PID Fuzzy Sliding Mode Controller", IJITCS, vol.6, no.2, pp.72-83, 2014. DOI: 10.5815/ijitcs.2014.02.10

[59] Kamran Heidari, Farzin Piltan, Samaneh Zahmatkesh, Sara Heidari, Mahdi Jafari,"Design High Efficiency Intelligent Robust Backstepping Controller ", IJIEEB, vol.5, no.6, pp.22-32, 2013. DOI: 10.5815/ijieeb.2013.06.03.

[60] Zahra Esmaieli, Farzin Piltan, Meysam Kazeminasab, Ali Reza Salehi, Mahdi Mirshekaran,"Design Intelligent Robust Back stepping Controller", IJMECS, vol.6, no.1, pp.53-64, 2014.DOI: 10.5815/ijmecs.2014.01.06

[61] Omid Avatefipour, Farzin Piltan, Mahmoud Reza Safaei Nasrabad, Ghasem Sahamijoo, Alireza Khalilian,"Design New Robust Self Tuning Fuzzy Backstopping Methodology", IJIEEB, vol.6, no.1, pp.49-61, 2014. DOI: 10.5815/ijieeb.2014.01.06

[62] Alireza Siahbazi, Ali Barzegar, Mahmood Vosoogh, Abdol Majid Mirshekaran, Samira Soltani,"Design Modified Sliding Mode Controller with Parallel Fuzzy Inference System Compensator to Control of Spherical Motor", IJISA, vol.6, no.3, pp.12-25, 2014. DOI: 10.5815/ijisa.2014.03.02

[63] Narges Gholami Mozafari, Farzin Piltan, Mohammad Shamsodini, Azita Yazdanpanah, Ali Roshanzamir,"On Line Tuning Premise and Consequence FIS Based on Lyaponuv Theory with Application to Continuum Robot", IJISA, vol.6, no.3, pp.96-110, 2014. DOI: 10.5815/ijisa.2014.03.10.

[64] Mansour Bazregar, Farzin Piltan, AliReza Nabaee, MohammadMahdi Ebrahimi,"Design Modified Fuzzy PD Gravity Controller with Application to Continuum Robot", IJITCS, vol.6, no.3, pp.82-94, 2014. DOI: 10.5815/ijitcs.2014.03.10

[65] Arzhang Khajeh, Farzin Piltan, Mohammad Reza Rashidian, Afsaneh Salehi, Ehsan pouladi ,"Design New Intelligent PID like Fuzzy Backstepping Controller ", IJMECS, vol.6, no.2, pp.15-26, 2014.DOI: 10.5815/ijmecs.2014.02.03

\section{Authors' Profiles}

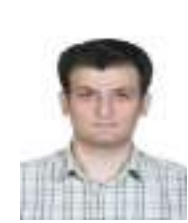

Alireza Khalilian is currently working as a primary researcher in the laboratory of Control and Robotic, Institute of Advance Science and Technology, IRAN SSP research and development Center. His current research interests are in the area of nonlinear control, artificial control system and robotics.

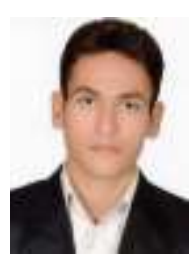

Ghasem Sahamijoo is currently working as a primary researcher in the laboratory of Control and Robotic, Institute of Advance Science and Technology, IRAN SSP research and development Center. His current research interests are in the area of nonlinear control, artificial control system and robotics.

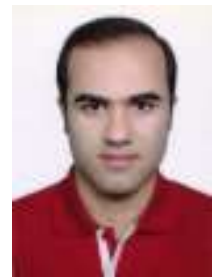

Omid Avatefipour is currently working as a primary researcher in the laboratory of Control and Robotic, Institute of Advance Science and Technology, IRAN SSP research and development Center. His current research interests are in the area of nonlinear control, artificial control system and robotics.

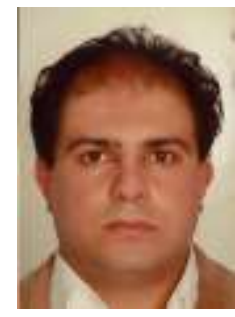

Farzin Piltan was born on 1975, Shiraz, Iran. In 2004 he is jointed Institute of Advance Science and Technology, Research and Development Center, IRAN SSP. Now he is a dean of Intelligent Control and Robotics Lab. In addition to 7 textbooks, Farzin Piltan is the main author of more than 100 scientific papers in refereed journals. He is editorial review board member for 'international journal of control and automation (IJCA), Australia, ISSN: 2005-4297; 'International Journal of Intelligent System and Applications (IJISA)', Hong Kong, ISSN:2074-9058; 'IAES international journal of robotics and automation, Malaysia, ISSN:2089-4856; 'International Journal of Reconfigurable and Embedded Systems', Malaysia, ISSN:2089-4864. His current research interests are nonlinear control, artificial control system and applied to FPGA, robotics and artificial nonlinear control and IC engine modeling and control.

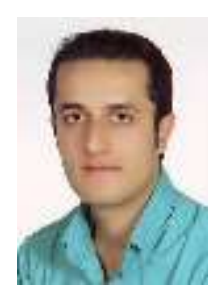

Mahmoud Reza Safaei Nasrabad is currently working as a primary researcher in the laboratory of Control and Robotic, Institute of Advance Science and Technology, IRAN SSP research and development Center. His current research interests are in the area of nonlinear control, artificial control system and robotics.

How to cite this paper: Alireza Khalilian, Ghasem Sahamijoo, Omid Avatefipour, Farzin Piltan, Mahmoud Reza Safaei Nasrabad,"Design High Efficiency-Minimum Rule Base PID Like Fuzzy Computed Torque Controller", International Journal of Information Technology and Computer Science(IJITCS), vol.6, no.7, pp.77-87, 2014. DOI: 10.5815/ijitcs.2014.07.10 